\title{
Intestinal endometriosis in a 36-year-old woman
}

\author{
Yasuhiko Hamada MD PhD, Kyosuke Tanaka MD PhD
}

Cite as: CMAJ 2020 August 17;192:E960. doi: 10.1503/cmaj.191471
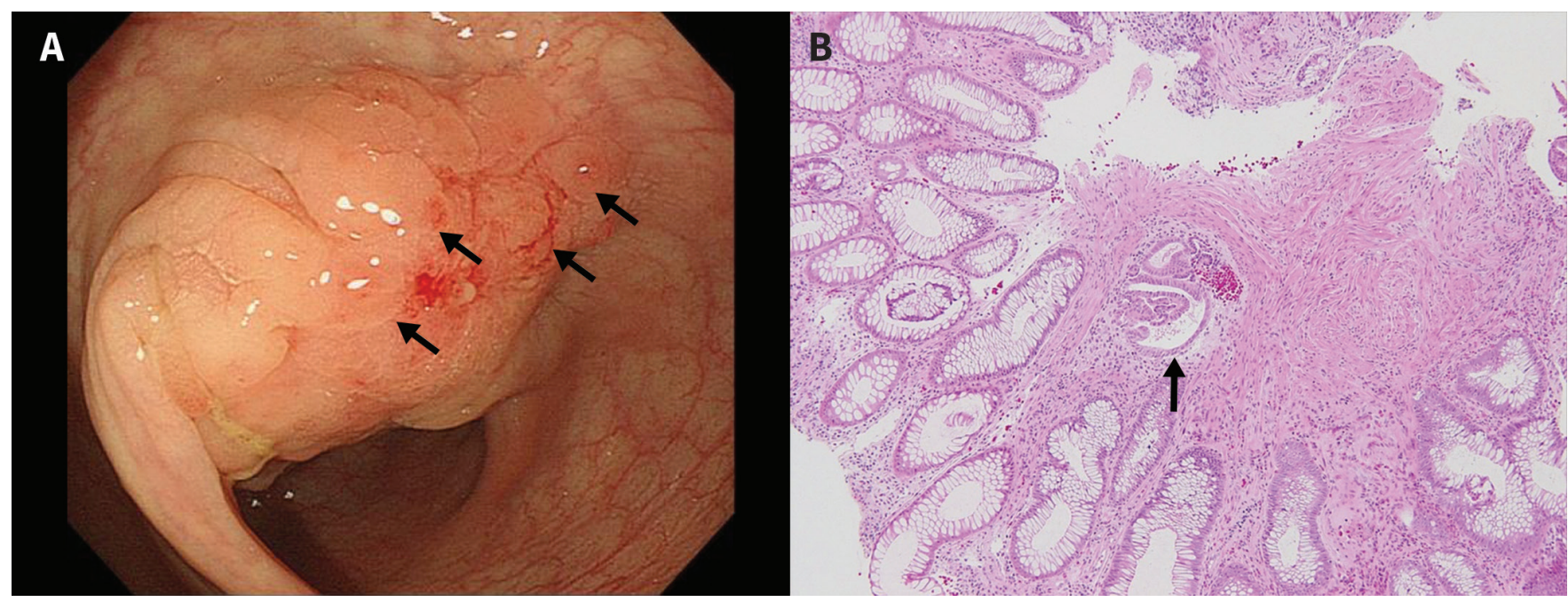

Figure 1: (A) Endoscopy image showing reddish nodules (arrows) atop a $30 \mathrm{~mm}$ submucosal lesion in the sigmoid colon of a 36-year-old woman. (B) Biopsy image obtained just before the patient's menstrual phase, showing a gland and stroma of endometrium (arrow) (hematoxylin and eosin stain; original magnification $\times 100$.

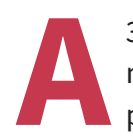

36-year-old woman presented to the emergency department with a 9-month history of abdominal pain accompanied by rectal bleeding during menstruation. She was otherwise well. Her pelvic and rectovaginal examination, as well as findings on transvaginal ultrasound, were unremarkable. $T_{2}$-weighted magnetic resonance imaging (MRI) showed a hypointense wall thickening in the sigmoid colon. We suspected endometriosis and our differential diagnosis included inflammatory bowel disease and cancer. Colonoscopy showed a 30-mm submucosal lesion with a red, nodular surface in the sigmoid colon (Figure 1A). Pathologic examination of biopsy specimens was nonspecific. Subsequently, biopsies performed during a second colonoscopy just before the patient's menstrual phase showed an endometriotic gland and stroma, positive for estrogen receptor (Figure 1B). We diagnosed intestinal endometriosis. Our patient preferred to avoid long-term hormone therapy and accepted the risk associated with laparoscopic sigmoidectomy. Laparoscopic exploration showed no other endometriotic implants.

Intestinal endometriosis occurs in $5 \%-15 \%$ of women with endometriosis, and as was the case with our patient, about $20 \%$ of women with intestinal endometriosis do not have pelvic endometriosis. ${ }^{1}$ The rectosigmoid colon is involved in 3 out of 4 patients with intestinal endometriosis. Although MRI and transvaginal ultrasound are sensitive diagnostic modalities for intestinal endometriosis, definitive diagnosis requires a biopsy. Endoscopic biopsies frequently yield insufficient tissue because the mucosal involvement is sparse. ${ }^{2}$ We performed endoscopic biopsies just before the menstrual phase, when endometriotic tissue is at its peak. First-line treatment of endometriosis is hormonal, but surgery may be performed for severe intestinal stenosis or to accommodate a patient's preference. The rate of recurrence after surgery is about $10 \%{ }^{3}$

\section{References}

1. Rossini LG, Ribeiro PA, Rodrigues FC, et al. Transrectal ultrasound - Techniques and outcomes in the management of intestinal endometriosis. Endosc Ultrasound 2012;1:23-35.

2. Bong JW, Yu CS, Lee JL, et al. Intestinal endometriosis: diagnostic ambiguities and surgical outcomes. World J Clin Cases 2019;7:441-51.

3. Meuleman C, Tomassetti C, D'Hoore A, et al. Surgical treatment of deeply infiltrating endometriosis with colorectal involvement. Hum Reprod Update 2011;17:311-26.

\section{Competing interests: None declared.}

This article has been peer reviewed.

The authors have obtained patient consent.

Affiliations: Departments of Gastroenterology and Hepatology (Hamada), and Endoscopic Medicine (Tanaka), Mie University Hospital, Tsu, Japan

Correspondence to: Yasuhiko Hamada, y-hamada@clin.medic.mie-u.ac.jp 